\title{
Clinical Features and Organ System Dysfunction in Coronavirus Disease 2019 in China
}

\author{
Po Cui*, Siyu Zhang*, Yuemin Nan\# ${ }^{(}$, Rongqi Wang ${ }^{\#}$ \\ Department of Traditional and Western Medical Hepatology, Third Hospital of Hebei Medical University \& Hebei Key \\ Laboratory of Mechanism of Liver Fibrosis in Chronic Liver Disease, Shijiazhuang, China \\ Email: Cuipo@163.com, zhangsiyu9105@163.com, "nanyuemin@163.com, "wangrongqi@163.com
}

How to cite this paper: Cui, P., Zhang, S.Y., Nan, Y.M. and Wang, R.Q. (2020) Clinical Features and Organ System Dysfunction in Coronavirus Disease 2019 in China. Journal of Biosciences and Medicines, 8, 70-85.

https://doi.org/10.4236/jbm.2020.811007

Received: August 12, 2020

Accepted: November 9, 2020

Published: November 12, 2020

Copyright $\odot 2020$ by author(s) and Scientific Research Publishing Inc. This work is licensed under the Creative Commons Attribution International License (CC BY 4.0).

http://creativecommons.org/licenses/by/4.0/

\begin{abstract}
Objectives: To enhance understanding of COVID-19 in clinical characteristics, related organ system dysfunctions and therapies. Methods: We enrolled patients with COVID-19 admitted to two Chinese hospitals from 19 January 2020 to 15 March 2020 and collected demographic and clinical data. Results: We retrospectively enrolled 130 patients: 7 mild, 103 moderate, and 20 severe cases. Severe patients were older than mild/moderate patients (60.1 vs 52.9 years; $\mathrm{P}=0.028)$ and had more comorbidities $(85.0 \%$ vs $52.7 \%$; $\mathrm{p}=0.006)$. 116 patients $(89.2 \%)$ were 2019 -noval coronavirus RNA-positive with a median detectable time of 10 days. Obvious absorbed of pneumonia in chest imaging occurred in 20 days. The most common symptoms were fever $(\mathrm{n}=$ $87,66.9 \%)$, cough $(\mathrm{n}=74,56.9 \%)$, fatigue $(\mathrm{n}=73,56.2 \%)$, and chest tightness ( $\mathrm{n}=54,41.5 \%)$. The incidence of liver, kidney, heart, and coagulation dysfunction was $38.4 \%, 24.5 \%, 30.1 \%$, and $24.6 \%$, respectively. The most frequently used antiviral therapies were arbidol $(\mathrm{n}=64,49.2 \%)$, oseltamivir $(\mathrm{n}=$ 73, 56.2\%), and Lianhua Qingwen $(n=73,56.2 \%)$. All patients recovered being hospitalized with a median duration of 15 days. Conclusions: Middle-aged and elderly with comorbidities are more likely to be infected with COVID-19 and develop severe symptoms. The lung, liver, heart, kidney, and coagulation system all may sustain injury, especially in severe cases.
\end{abstract}

\section{Keywords}

Severe Acute Respiratory Syndrome Coronavirus 2, Coronavirus Disease 2019, Clinical Characteristics, Organ System Dysfunction, Therapy

\section{Introduction}

Novel coronavirus disease 2019 (COVID-19) is a viral respiratory illness caused

*These authors contributed equally to this work. 
by severe acute respiratory syndrome coronavirus 2 (SARS-CoV-2). COVID-19 emerged in Wuhan City in late December 2019 and spread rapidly throughout China [1]. Subsequently, cases have been reported in many other countries, and managing COVID-19 has become a global challenge regarding public health. COVID-19 was declared a pandemic by the World Health Organization on 11 March 2020. To date (23 April 2020), the epidemic is on-going in at least 200 countries, with over 2.7 million confirmed cases and more than 180,000 deaths worldwide [2]. Exactly how coronavirus (COVID-19) spreads from person to person is unknown, but similar viruses can spread through cough droplets, close contact, aerosols, and potentially, other routes [3]. The disease may progress rapidly and result in acute respiratory distress syndrome. Other organ-system dysfunctions (i.e., liver, kidney, cardiac, and coagulation systems) and death can occur in severe cases [4] [5] [6]. However, COVID-19-associated organ system dysfunction is known, but has not been reported in depth. Our objective in this study was to retrospectively describe the clinical characteristics, laboratory examination findings, radiological features, and related organ system dysfunctions of 130 hospitalized patients with COVID-19 symptoms at Wuhan Seventh Hospital and Jiangan mobile cabin hospitals in Wuhan, Hubei province, China and to compare severe patients with mild- and moderate patients.

\section{Methods}

\section{Participants and Sources}

We enrolled all patients with COVID-19 who were admitted to Jiangan mobile cabin hospitals or Wuhan Seventh Hospital from 15 February 2020 to 15 March 2020. The first author worked in the fight against COVID-19 as a front-line doctor in two hospitals successively. All patients met the Diagnosis and Treatment Protocol for Novel Coronavirus Pneumonia (Trial Version 7) released by the National Health Commission \& State Administration of Traditional Chinese Medicine, China, on 3 March 2020 [7]. The criteria including: 1) history of travel or residence in an epidemic area within 14 days before symptom onset; 2) contact history with patients confirmed with positive SARS-CoV-2 nucleic acid test or patients with fever or respiratory symptoms from an epidemic area within 14 days before symptom onset; 3) clustering of cases; 4) fever and/or respiratory symptoms; 5) imaging features of pneumonia; 6) normal or decreased total white blood cell count and decreased lymphocyte count early in the disease; 7) positive SARS-CoV-2 nucleic acid test by real-time fluorescence polymerase chain reaction testing (RT-PCR); 8) virus gene sequence highly homologous with the known SARS-CoV-2; and 9) SARS-CoV-2 specific Ig M and IgG are detectable in serum or IgG is detectable or reaches a titration of at least 4-fold increase during convalescence compared with the acute phase. Patients who satisfied any one of the epidemiological histories (criteria 1 - 3) as well as any two of the clinical manifestations (criteria 4 - 6) or three clinical manifestation with no definite epidemiological history were diagnosed as suspected cases. Suspected cases with one of 
the etiological criteria (criteria 7 - 9) were diagnosed as confirmed cases. Chest imaging of COVID-19 include: 1) small patchy shadows; 2) separated or mixed ground-glass opacities; 3) consolidation; 4) interstitial changes: vascular enlargement, interlobular septal thickening and reticulation. We excluded participants with the following conditions: 1) upper respiratory infections, viral pneumonia or mycoplasma pneumonia caused by other viruses, such as influenza virus, adenovirus or respiratory syncytial virus; 2) non-infectious diseases such as vasculitis, dermatomyositis, and organizing pneumonia. What's more, patients with typical chest imaging manifestations but without positive results of the nucleic acid test were also enrolled after assessing the exclusion criteria.

They were clinically classified as mild cases (mild clinical symptoms with no sign of pneumonia on imaging), moderate cases (showing fever and respiratory symptoms with radiological findings of pneumonia), severe cases (respiration $\geqq$ 30 breaths/min, oxygen saturation $\leq 93 \%$ at rest, arterial partial pressure of oxygen $\left(\mathrm{PaO}_{2}\right) /$ fraction of inspired oxygen $\left(\mathrm{FiO}_{2}\right) \leq 300 \mathrm{mmHg}$ or obvious lesion progression within $24-48$ hours $>50 \%$ in chest imaging) and critical cases (organ failures that requires alternative therapy and/or ICU care, or shock).

Liver injury was assessed according to the protocol for prevention, diagnosis, and treatment of liver injury in coronavirus disease 2019 and the Chinese diagnosis and treatment guidelines for drug-induced liver injury [8] [9]. We referred to the Kidney Disease: Improving Global Outcomes clinical practice guidelines for acute kidney injury and the Expert Recommendations for Clinical Management of Myocardial Injury Associated with Coronavirus Disease 2019 (First Edition), when we evaluated kidney and myocardial injuries, respectively [10] [11]. This study was reviewed and approved by the Human Ethics Committee, Third Hospital of Hebei Medical University (Approval number: 2020-013-1). Data were analyzed and interpreted by the author team.

\section{Data Collection}

We retrospectively collected patients' basic characteristics, namely epidemiological histories, clinical manifestations, and comorbidities on admission. We also evaluated patients' chest computed tomography (CT) images, SARS-CoV-2 RNA detection results, blood laboratory examination results, therapeutic interventions (i.e., antiviral therapy, corticosteroid therapy, traditional Chinese medicine therapies), and outcomes, from patients' electronic medical records, after admission. Serial monitoring of patients' laboratory profile was performed according to their clinical progression.

Oropharyngeal swab samples were collected for all patients in our study. SARS-CoV-2 nucleic acid was detected with fluorescence RT-PCR assay according to the manufacturer's protocol (Shanghai BioGerm Medical Technology Co., Ltd., Shanghai, China). Testing to detect 2019-nCoV RNA was performed on admission, 3 days after the disappearance of symptoms, and 24 hours after negative RNA seroconversion. All patients must have had at least three consecutive 
negative RNA results to be considered recovered. We used standard lung window and mediastinal window views in chest imaging using dual-source CT for radiographic assessments. Chest $\mathrm{CT}$ was performed on admission and after two negative 2019-nCOV RNA detection test results. The frequency of chest CT scans was according to severity and dynamic changes of the disease.

\section{Statistical Analysis}

Continuous variables were described as median (interquartile range) or mean \pm standard deviation and categorical variables as frequencies or percentages (\%). All data were analyzed using SPSS version 26.0 (IBM Corp., Armonk, NY). We used the independent samples $t$ test to compare the means between two groups and the Mann-Whitney test to compare medians. Frequencies and percentages were compared using the chi-squared test or Fisher's exact test, conditionally. p-values $<0.05$ were considered statistically significant.

\section{Results}

\subsection{Patients' Basic Characteristics}

Of the 130 patients with COVID-19, 7 patients were mild cases, 103 were moderate, and 20 were severe. Patients' median age was 54 years (range: 16 - 87 years) (Figure 1), and severe patients were older than the mild/moderate patients $(52.9 \pm 13.1$ years vs $60.1 \pm 12.1$ years, $\mathrm{p}=0.028)$. There was a slight but non-significant difference in the prevalence in men between the severe and mild/moderate groups ( $65.0 \%$ vs $46.4 \%, \mathrm{p}=0.098)$. Seventy-five patients $(57.7 \%)$ had comorbidities, namely hypertension $(\mathrm{n}=31)$, type 2 diabetes mellitus $(\mathrm{n}=$ 18), chronic liver disease $(\mathrm{n}=10)$, and coronary heart disease/arrhythmia $(\mathrm{n}=$ 11). Chronic live disease included nonalcoholic fatty liver disease $(n=5)$, chronic hepatitis $B(n=4)$ and autoimmune hepatitis $(n=1)$. Additionally, 31 patients had chronic gastritis $(\mathrm{n}=9)$, prior cerebral infarction $(\mathrm{n}=4)$, thyroid dysfunction $(\mathrm{n}=$ 5) and hyperuricemia/gout $(n=4)$ and so on. It is important to point out that $85.0 \%$ of severe patients had comorbidities, and the percentage was significantly higher than that of mild/moderate patients $(\mathrm{p}=0.006)$ (Table 1$)$.

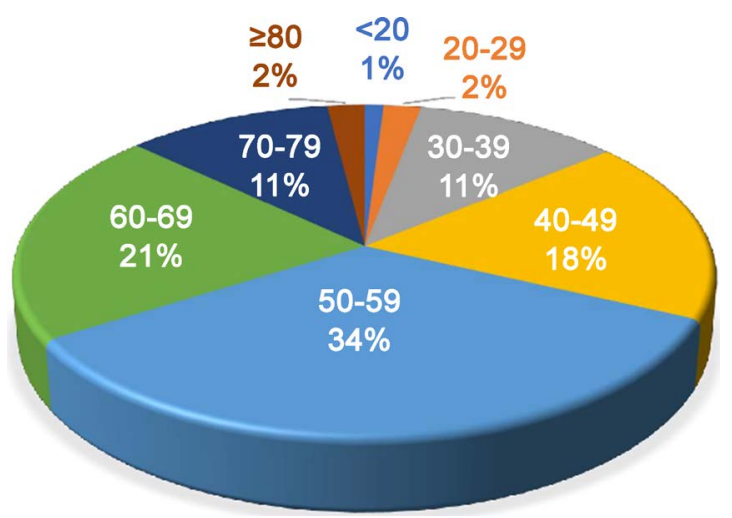

Figure 1. Age distribution of the 130 patients with COVID-19 in this study. 
Table 1. Basic characteristics of the patients with COVID-19.

\begin{tabular}{|c|c|c|c|c|}
\hline Characteristics (n, \%) & $\begin{array}{c}\text { Total } \\
(n=130)\end{array}$ & $\begin{array}{l}\text { Mild/moderate } \\
(\mathrm{n}=110)\end{array}$ & $\begin{array}{c}\text { Severe } \\
(\mathrm{n}=20)\end{array}$ & $P$ \\
\hline Male & $64(49.2)$ & $51(46.4)$ & $13(65.0)$ & 0.098 \\
\hline Age, $M D \pm S D, y$ & $54.4 \pm 13.1$ & $52.9 \pm 13.1$ & $60.1 \pm 12.1$ & 0.028 \\
\hline \multicolumn{5}{|l|}{ Comorbidities } \\
\hline Hypertension & $31(23.8)$ & $23(20.9)$ & $8(40.0)$ & 0.064 \\
\hline Diabetes & $18(13.8)$ & $12(10.9)$ & $6(30.0)$ & 0.034 \\
\hline Coronary heart disease & $11(8.5)$ & $7(6.4)$ & $4(20.0)$ & 0.066 \\
\hline Chronic liver disease & $10(7.7)$ & $8(7.3)$ & $2(10.0)$ & 0.475 \\
\hline Chronic lung disease & $6(4.6)$ & $4(3.6)$ & $2(10.0)$ & 0.230 \\
\hline Other & $31(23.8)$ & $25(22.7)$ & $6(30.0)$ & 0.328 \\
\hline None & $55(42.3)$ & $52(47.3)$ & $3(15.0)$ & 0.006 \\
\hline \multicolumn{5}{|l|}{ Symptoms } \\
\hline Fever & $87(66.9)$ & $69(62.7)$ & $18(90.0)$ & 0.012 \\
\hline Cough & $74(56.9)$ & $58(52.7)$ & $16(80.0)$ & 0.019 \\
\hline Fatigue & $73(56.2)$ & $58(52.7)$ & $15(75.0)$ & 0.052 \\
\hline Chest tightness & $54(41.5)$ & $42(38.2)$ & $12(60.0)$ & 0.058 \\
\hline Diarrhea & $38(29.2)$ & $35(31.8)$ & $3(15.0)$ & 0.101 \\
\hline Anorexia & $37(28.5)$ & $29(26.4)$ & $8(40.0)$ & 0.165 \\
\hline Pharyngalgia & $16(12.3)$ & $16(14.5)$ & 0 & 0.070 \\
\hline Headache & $14(10.8)$ & $14(12.7)$ & 0 & 0.092 \\
\hline Myalgia & $8(6.2)$ & $6(5.5)$ & $2(10.0)$ & 0.356 \\
\hline Asymptomatic & $10(7.7)$ & $9(8.2)$ & $1(5.0)$ & 0.086 \\
\hline Positive CT and RT-PCR & $109(83.8)$ & $92(83.6)$ & $17(85.0)$ & 0.590 \\
\hline Positive CT only & $14(10.8)$ & $11(10.0)$ & $3(15.0)$ & 0.368 \\
\hline RNA negative conversion, $\mathrm{d}$ & $11.0(6.0,14.3)$ & $11.0(6.0,14.0)$ & $11.0(2.8,15.3)$ & 0.672 \\
\hline Obvious improvement of CT, $\mathrm{d}$ & $19.8 \pm 8.8$ & $19.3 \pm 8.7$ & $22.0 \pm 8.9$ & 0.219 \\
\hline \multicolumn{5}{|l|}{ Antiviral therapy } \\
\hline Oseltamivir & $60(46.2)$ & $52(47.3)$ & $8(40.0)$ & 0.550 \\
\hline Ribavirin & $24(18.5)$ & $18(16.4)$ & $6(30.0)$ & 0.150 \\
\hline Abidor & $64(49.2)$ & $59(53.6)$ & $5(25.0)$ & 0.019 \\
\hline Lianhua Qingwen & $73(56.2)$ & $67(60.9)$ & $6(30.0)$ & 0.011 \\
\hline Peginterferon alfa- $1 \mathrm{~b}$ & $7(5.4)$ & $4(3.6)$ & $3(15.0)$ & 0.039 \\
\hline Azithromycin & $29(22.3)$ & $20(18.2)$ & $9(45.0)$ & 0.008 \\
\hline Moxifloxacin & $81(62.3)$ & $66(60.0)$ & $15(75.0)$ & 0.153 \\
\hline Other antibiotics & $29(22.3)$ & $23(20.9)$ & $6(30.0)$ & 0.265 \\
\hline Chinese herbal decoction & $48(36.9)$ & $31(28.2)$ & $17(85.0)$ & 0.000 \\
\hline Hospital stay, d & $15.0(10.3,23.0)$ & $12.5(8.3,18.8)$ & $23.0(16.0,28.8)$ & 0.000 \\
\hline
\end{tabular}

Others: Chronic gastritis, Prior cerebral infarction, Thyroid dysfunction, Hyperuricemia/Gout. MD, mean; $\mathrm{SD}$, standard deviation; CT, computed tomography; RT-PCR, real-time polymerase chain reaction; RNA, ribonucleic acid; d, day. 
The interval between exposure to patients or epidemic areas and the onset of fever and/or cough ranged from 2 to 14 days. Of the 130 patients, the most common symptoms at presentation were fever $(\mathrm{n}=87,66.9 \%)$, cough $(\mathrm{n}=74$, $56.9 \%)$, fatigue ( $\mathrm{n}=73,56.2 \%)$, and chest tightness $(\mathrm{n}=54,41.5 \%)$. Less common symptoms included diarrhea $(n=38,29.2 \%)$, anorexia $(n=37,28.5 \%)$, sore throat $(\mathrm{n}=16,12.3 \%)$, headache $(\mathrm{n}=14,10.8 \%)$, and runny nose $(\mathrm{n}=3,2.3 \%)$ (Table 1). Patients' median body temperature was $38.2^{\circ} \mathrm{C}$ and ranged from $36.2^{\circ} \mathrm{C}$ to $39.5^{\circ} \mathrm{C}$. Rash, purpura, and lymphadenopathy were not seen in this cohort.

\subsection{Family Medical History and Potential Route of Transmission}

In the 130 patients, 128 individuals were permanent residents of Wuhan, and the remaining two patients came from Huanggang City and Jinan City, respectively. Fifty-seven (43.8\%) patients had infected family members, indicating that person-to-person contact among family members was likely the main transmission routine (Table 1$)$.

\subsection{Clinical Manifestations}

\subsubsection{Detection of 2019-nCoV RNA and Typical CT Imaging Features}

Admission of the patient depended on their epidemiological history, clinical manifestations, positive 2019-nCoV RNA detection, and chest CT imaging findings typical of COVID-19. All patients underwent oropharyngeal swab sample collection to detect 2019-nCoV RNA, and chest CT scans. Overall, 108 (83.1\%) patients had both positive detection of 2019-nCoV RNA and typical chest CT imaging features of COVID-19 at admission. One patient showed only typical chest CT imaging findings for COVID-19 at admission, but had a 2019-nCOV RNA-positive result 4 days after admission. Fourteen (10.8\%) patients had only typical chest CT features during hospitalization and negative or suspected results for RNA detection throughout hospitalization. The duration of continuously-detectable viral RNA in 116 patients ranged from 1 to 41 days, with a median of 10 days. There was no significant difference in duration between severely and non-severely affected patients. Of the 123 patients with abnormal chest radiographic findings, parenchymal abnormalities in the peripheral zone was the most common finding. Airspace ground-glass opacities, consolidation, or mixed ground-glass opacities developed in different patients during the course of the disease (Figure 2). Successful response to therapy was demonstrated by serial chest radiographs, which showed resolution of lung inflammation. The mean time to resolution or obvious absorbed of pneumonia was $19.5 \pm 8.9$ days, which was slightly but not significantly longer in severe vs non-severe patients $(22.0 \pm$ 8.9 days vs $19.3 \pm 8.7$ days, $\mathrm{p}=0.219$ ) (Table 1$)$.

\subsubsection{Hematological Findings}

Higher white blood cell and neutrophil counts $\left(6.5 \times 10^{9} / \mathrm{L}\right.$ vs $4.8 \times 10^{9} / \mathrm{L}, \mathrm{p}=$ 0.003 and $4.6 \times 10^{9} / \mathrm{L}$ vs $3.3 \times 10^{9} / \mathrm{L}, \mathrm{p}=0.000$, respectively). In contrast, lymphocyte counts were lower (median: $0.7 \times 10^{9} / \mathrm{L}$ vs $1.2 \times 10^{9} / \mathrm{L}, \mathrm{p}=0.001$ ). Red blood 
cell counts and platelet counts were normal in both groups. There were significant differences in erythrocyte sedimentation rate $(20.0 \mathrm{~mm} / \mathrm{h}$ vs $12.5 \mathrm{~mm} / \mathrm{h}, \mathrm{p}=0.011)$, C-reactive protein $(37.5 \mathrm{mg} / \mathrm{mL}$ vs $12.2 \mathrm{mg} / \mathrm{mL}, \mathrm{p}=0.025)$, and serum amyloid A (103.6 mg/L vs $23.7 \mathrm{mg} / \mathrm{L}, \mathrm{p}=0.017$ ) between severe and mild/moderate patients, respectively, indicating a systemic inflammatory response in the severe patients. Fasting blood glucose level, as a metabolic index, was markedly higher in severe patients $(8.5 \mathrm{mmol} / \mathrm{L}$ vs $5.5 \mathrm{mmol} / \mathrm{L}, \mathrm{p}=0.000)$, echoing the higher prevalence of type 2 diabetes mellitus in the group (30.0\% vs $10.9 \%, \mathrm{p}=0.034)$ (Table 1 and Table 2).

\subsection{Clinical Analysis of the Extrapulmonary Manifestations}

\subsubsection{Liver Injury}

The general incidence of liver injury was $21.5 \%(28 / 130), 13.6 \%(15 / 110)$ in
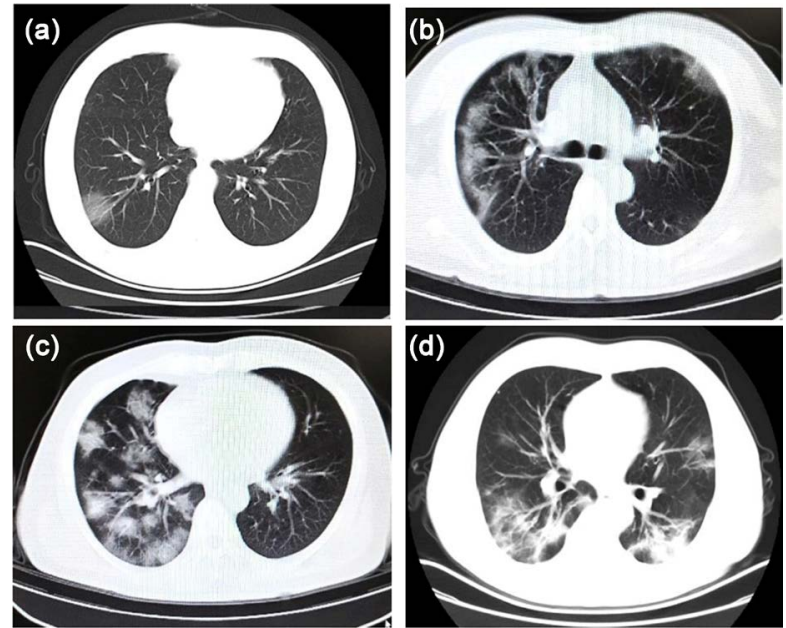

Figure 2. Typical features of COVID-19 in chest computed tomography images: (a) Airspace ground-glass opacities in the peripheral zone, (b) Mixed ground-glass opacities in the peripheral zone, (c) Mixed ground-glass opacities in the right lung, and (d) Consolidation.

Table 2. Comparison of routine blood examination results and inflammatory markers between the two groups.

\begin{tabular}{cccc}
\hline Variables & $\begin{array}{c}\text { Mild/moderate } \\
(\mathbf{n}=110)\end{array}$ & Severe $(\mathbf{n}=\mathbf{2 0})$ & $P$ \\
\hline White blood cell count $\times 10^{9} / \mathrm{L}$ & $4.8(4.0,6.1)$ & $6.5(5.1,8.3)$ & 0.003 \\
Neutrophil count $\times 10^{9} / \mathrm{L}$ & $3.3(2.3,3.8)$ & $4.6(3.8,6.8)$ & 0.000 \\
Lymphocyte count $\times 10^{9} / \mathrm{L}$ & $1.2(0.9,1.7)$ & $0.7(0.5,1.1)$ & 0.001 \\
Hemoglobin $\times \mathrm{g} / \mathrm{L}$ & $124.2 \pm 16.6$ & $125.2 \pm 13.0$ & 0.817 \\
Platelet count $\times 10^{9} / \mathrm{L}$ & $213.9 \pm 71.0$ & $213.1 \pm 94.8$ & 0.965 \\
Procalcitonin $\times \mathrm{ng} / \mathrm{mL}$ & $0.04(0.03,0.07)$ & $0.06(0.04,0.13)$ & 0.066 \\
C-reactive protein $\times \mathrm{mg} / \mathrm{L}$ & $12.2(1.6,50.1)$ & $37.5(12.4,130.1)$ & 0.025 \\
Serum amyloid $\mathrm{A} \times \mathrm{mg} / \mathrm{L}$ & $23.7(6.6,96.2)$ & $103.6(35.2,152.8)$ & 0.017 \\
Erythrocyte sedimentation $\mathrm{rate} \times \mathrm{mm} / \mathrm{h}$ & $12.5(6.3,25.8)$ & $28.0(21.0,33.5)$ & 0.011 \\
\hline
\end{tabular}


mild/moderate patients and $65.0 \%(13 / 20)$ in severe patients. Seventeen of them had comorbidities and two were chronic liver disease. The incidence of liver injury in patients with comorbidities was $22.7 \%(17 / 75)$ and $20.0 \%(2 / 10)$ in patients with chronic liver disease. No significant difference was observed in the incidence between patients with and without comorbidities or chronic liver disease $(22.7 \%$ vs $20.0 \%$ and $20.0 \%$ vs $21.7 \%$, respectively, $p>0.05)$. The injury in majority ( $\mathrm{n}=23,82.1 \%$ ) were mild. Only five patients had increased alanine aminotransferase (ALT) or aspartate aminotransferase (AST) levels $>3$ times of the upper limit of normal (ULN). Especially, ALT and AST levels in one patient who had taken four medications (arbidol, oseltamivir, Lianhua Qingwen and moxifloxacin) for more than 5 days, were $983 \mathrm{IU} / \mathrm{L}$ and $380 \mathrm{IU} / \mathrm{L}$, respectively, during hospitalization. No significant difference existed in the severity of liver injury between patients with and without comorbidities (Table 3). Furthermore, liver injury occurred before admission in 17 (60.7\%) patients and after admission in $11(39.3 \%)$ patients with no significant difference in the severity (Table 4). Liver injury in the former did not progress further after admission. The time point of liver injury occurred in the latter was about 10 days (Figure 3 ).

\subsubsection{Kidney Injury}

Mild kidney injury occurred in 13 (10.0\%) patients: 7 (35.0\%) were severe and 6 (5.5\%) were non-severe cases. The mean age of affected patients was $63.0 \pm 11.5$

Table 3. Albumin and liver enzyme levels in patients with COVID-19-related liver injury, according to the presence of comorbidities.

\begin{tabular}{|c|c|c|c|}
\hline & $\begin{array}{c}\text { Absence of } \\
\text { Comorbidities } \\
(\mathrm{n}=11)\end{array}$ & $\begin{array}{c}\text { Presence of } \\
\text { comorbidities } \\
(n=17)\end{array}$ & $P^{\star}$ \\
\hline Age, $M D \pm S D, y$ & $55.3 \pm 9.2$ & $61.4 \pm 10.6$ & 0.128 \\
\hline Male, $n,(\%)$ & $5(45.5)$ & $11(64.7)$ & 0.441 \\
\hline \multicolumn{4}{|l|}{$\operatorname{Albumin}(\mathrm{g} / \mathrm{L})$} \\
\hline baseline & $36.5 \pm 3.8$ & $35.3 \pm 4.8$ & 0.430 \\
\hline extremum & $32.7 \pm 4.6$ & $30.5 \pm 5.3$ & 0.254 \\
\hline$P^{*}$ & 0.047 & 0.010 & \\
\hline \multicolumn{4}{|c|}{ Alanine aminotransferase $\times \mathrm{U} / \mathrm{L}$} \\
\hline baseline & $33.0(21.0,63.0)$ & $39.0(27.3,60.0)$ & 0.677 \\
\hline extremum & $81.0(54.0,137.0)$ & $78.0(48.5,109.5)$ & 0.285 \\
\hline$P^{*}$ & 0.013 & 0.009 & \\
\hline \multicolumn{4}{|c|}{ Aspartate aminotransferase $\times \mathrm{U} / \mathrm{L}$} \\
\hline baseline & $42.4 \pm 16.2$ & $49.0 \pm 21.8$ & 0.375 \\
\hline extremum & $62.0(41.0,92.0)$ & $57.0(48.0,68.5)$ & 0.175 \\
\hline$P^{*}$ & 0.023 & 0.274 & \\
\hline
\end{tabular}

$P^{\star}$ : comparing albumin and liver enzyme levels according to the presence of comorbidities; $P^{\sharp}$ : comparing liver enzyme levels between baseline and the subsequently increased values. 
Table 4. Albumin and liver enzyme levels in patients with COVID-19-related liver injury occurring at or after admission.

\begin{tabular}{cccc}
\hline & $\begin{array}{c}\text { Occurred at } \\
\text { admission }(\mathrm{n}=17)\end{array}$ & $\begin{array}{c}\text { Occurred after } \\
\text { admission }(\mathrm{n}=11)\end{array}$ & $\boldsymbol{P}^{*}$ \\
\hline Age, MD \pm SD, y & $61.4 \pm 9.8$ & $55.4 \pm 10.5$ & 0.138 \\
Male, $\mathrm{n},(\%)$ & $9(52.9)$ & $7(63.6)$ & 0.054 \\
Albumin (g/L) & & & \\
baseline & $35.9 \pm 4.3$ & $35.4 \pm 4.6$ & 0.802 \\
extremum & $31.8 \pm 5.3$ & $30.7 \pm 4.8$ & 0.591 \\
$P^{\sharp}$ & 0.019 & 0.028 & \\
Alanine aminotransferase $\times \mathrm{U} / \mathrm{L}$ & & & 0.000 \\
baseline & $56.0(38.0,89.0)$ & $21.0(15.0,29.0)$ & 0.677 \\
extremum & $66.0(47.0,126.0)$ & $81.0(68.0,106.0)$ & \\
$P^{\sharp}$ & 0.333 & 0.000 & 0.000 \\
Aspartate aminotransferase $\times \mathrm{U} / \mathrm{L}$ & & & 0.225 \\
baseline & $55.0(42.0,70.0)$ & $29.0(21.0,35.0)$ & \\
extremum & $64.0(47.0,78.5)$ & $54.0(46.0,62.0)$ & \\
$P^{\sharp}$ & 1.000 & 0.004 & \\
\hline
\end{tabular}

$P:$ comparing albumin and liver enzyme levels between liver injury occurring at and after admission; $P^{\sharp}$ : comparing liver enzyme levels between baseline and the subsequently increased values.
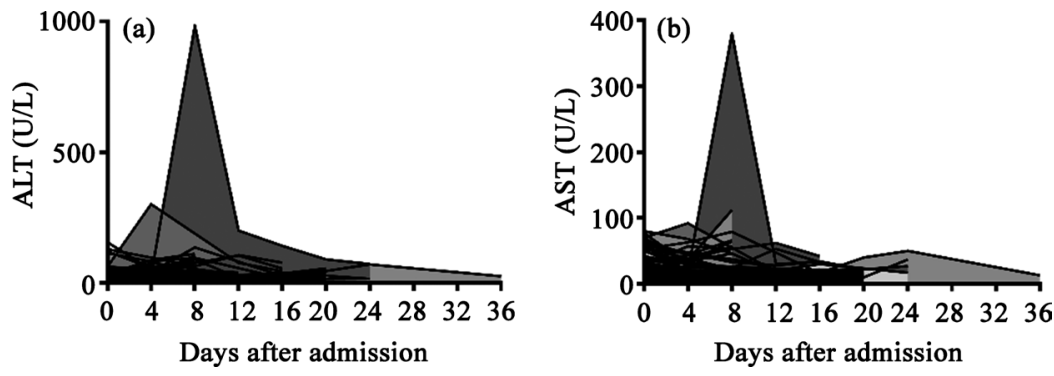

Figure 3. Dynamic changes of transaminase levels in the 28 patients with liver injury; (a) alanine transaminase, and (b) aspartate aminotransferase.

years, and 7 (53.8\%) patients were men. Seven of them also had hypertension and diabetes at baseline. Serum creatinine levels were markedly higher in severe patients than in non-severe patients $(75.0 \mu \mathrm{mol} / \mathrm{L}$ vs $57.0 \mu \mathrm{mol} / \mathrm{L}, \mathrm{p}=0.027)$, while the estimated glomerular filtration rate showed a converse change in severe vs non-severe cases $\left(95 \mathrm{ml} / \mathrm{min} / 1.72 \mathrm{~m}^{2}\right.$ vs $\left.123 \mathrm{ml} / \mathrm{min} / 1.72 \mathrm{~m}^{2}, \mathrm{p}=0.020\right)$ (Table 5).

\subsubsection{Myocardial Injury}

Twenty-two (16.9\%) patients developed myocardial injury associated with COVID-19. The average age of the 22 patients was $66.4 \pm 8.5$ years: 13 (59.1\%) men and $9(40.9 \%)$ women. The levels of myocardial injury markers, such as creatine kinase- $\mathrm{MB}$, myoglobin, cardiac troponin $\mathrm{T}$, and $\mathrm{N}$-terminal B-type 
Table 5. Biochemistry examination results for the main organ systems between the two groups.

\begin{tabular}{|c|c|c|c|}
\hline Variables & $\begin{array}{l}\text { Mild/moderate } \\
\quad(\mathrm{n}=110)\end{array}$ & $\begin{array}{l}\text { Severe } \\
(n=20)\end{array}$ & $P$ \\
\hline \multicolumn{4}{|l|}{ Liver } \\
\hline Albumin $\times \mathrm{g} / \mathrm{L}$ & $39.3 \pm 5.2$ & $34.3 \pm 4.3$ & 0.001 \\
\hline$<30 \mathrm{~g} / \mathrm{L}(\mathrm{n}, \%)$ & $6(11.3)$ & $12(60.0)$ & 0.000 \\
\hline Alanine aminotransferase $\times \mathrm{U} / \mathrm{L}$ & $19.0(14.0,32.0)$ & $30.5(23.3,53.8)$ & 0.015 \\
\hline Aspartate aminotransferase $\times \mathrm{U} / \mathrm{L}$ & $23.0(18.5,32.0)$ & $39.5(26.8,57.3)$ & 0.002 \\
\hline Total bilirubin $\times \mathrm{mmol} / \mathrm{L}$ & $6.7(2.7,9.8)$ & $7.1(4.4,9.7)$ & 0.255 \\
\hline \multicolumn{4}{|l|}{ Cardiac } \\
\hline Creatine kinase- $\mathrm{MB} \times \mathrm{ng} / \mathrm{ml}$ & $0.9(0.7,1.5)$ & $2.8(1.2,4.1)$ & 0.001 \\
\hline Myoglobin $\times \mathrm{ng} / \mathrm{ml}$ & $24.9(21.0,38.3)$ & $59.5(26.9,118.0)$ & 0.001 \\
\hline Cardiac troponin $\mathrm{T} \times \mathrm{ng} / \mathrm{ml}$ & $0.007(0.005,0.010)$ & $0.012(0.007,0.020)$ & 0.021 \\
\hline $\begin{array}{l}\text { N-terminal B-type natriuretic peptide } \\
\text { precursor } \times \mathrm{pg} / \mathrm{ml}\end{array}$ & $85.2(35.9,184.7)$ & $266.8(113.8,422.0)$ & 0.003 \\
\hline \multicolumn{4}{|l|}{ Kidney } \\
\hline Urea nitrogen $\times \mathrm{mmol} / \mathrm{L}$ & $4.2(3.3,5.2)$ & $5.0(3.7,7.6)$ & 0.061 \\
\hline serum creatinine $\times \mu \mathrm{mol} / \mathrm{L}$ & $57.0(51.0,71.0)$ & $75.0(55.8,86.8)$ & 0.027 \\
\hline Estimated glomerular filtration rate $\times \mathrm{ml} / \mathrm{min}$ & $123.0(108.0,142.0)$ & $95.0(77.8,122.5)$ & 0.020 \\
\hline Serum sodium $\times \mathrm{mmol} / \mathrm{L}$ & $141.4 \pm 2.6$ & $139.4 \pm 4.1$ & 0.071 \\
\hline Serum potassium serum $\times \mathrm{mmol} / \mathrm{L}$ & $3.8 \pm 0.4$ & $3.7 \pm 0.7$ & 0.390 \\
\hline \multicolumn{4}{|l|}{ Coagulation } \\
\hline Prothrombin time $\times s$ & $13.1(11.3,15.6)$ & $13.8(12.2,15.7)$ & 0.286 \\
\hline D-Dimer $\times \mu \mathrm{g} / \mathrm{ml}$ & $0.1(0.1,0.2)$ & $0.5(0.1,1.3)$ & 0.003 \\
\hline \multicolumn{4}{|l|}{ Metabolism } \\
\hline Total cholesterol $\times \mathrm{mmol} / \mathrm{L}$ & $3.8(2.9,4.5)$ & $3.2(2.8,3.7)$ & 0.133 \\
\hline Total triglyceride $\times \mathrm{mmol} / \mathrm{L}$ & $0.8(0.6,1.3)$ & $0.9(0.6,1.1)$ & 0.868 \\
\hline Fasting blood glucose $\times \mathrm{mmol} / \mathrm{L}$ & $5.5(5.2,6.5)$ & $8.5(5.9,11.8)$ & 0.000 \\
\hline
\end{tabular}

natriuretic peptide precursor, were increased, especially in severe patients (Table 5). The incidence of COVID-19-related myocardial injury was significantly higher in severe patients than in mild/moderate patients $(45.0 \%$ vs $11.8 \%, \mathrm{p}=$ 0.001). However, no patients with myocardial injury developed heart failure, malignant arrhythmia, or cardiogenic shock.

\subsubsection{Coagulation Function}

Prothrombin time remained normal in most patients. Coagulation dysfunction manifested mainly as increased D-dimer levels, which were increased in 32 (24.6\%) patients; 13 severe patients and 19 mild/moderate patients. D-dimer levels were markedly higher in severely affected patients than in non-severe patients (median, $0.5 \mu \mathrm{g} / \mathrm{ml}$ vs $0.1 \mu \mathrm{g} / \mathrm{ml}, \mathrm{p}=0.003$ ) (Table 5 ). No affected patients 
progressed to obvious blood clotting and bleeding disorders.

\subsection{Treatment and Clinical Outcomes}

Routine doses of antiviral drugs supplemented by symptomatic supportive therapy and traditional Chinese herbal remedies were used in the management of COVID-19. The most common antiviral drugs were arbidol $(\mathrm{n}=64,49.2 \%)$, oseltamivir ( $\mathrm{n}=73,56.2 \%)$, and the Chinese remedy Lianhua Qingwen $(\mathrm{n}=73$, $56.2 \%)$. Ribavirin $(\mathrm{n}=24,18.5 \%)$ and peginterferon alfa- $1 \mathrm{~b}(7,5.4 \%)$ were also used in some patient. The duration of antiviral treatment was no more than 10 days. Antibiotic medication was also administered for either prevention or treatment of bacterial infections, and constituted moxifloxacin ( $\mathrm{n}=81,62.3 \%)$ and azithromycin $(n=29,22.3 \%)$. Single or combined antibiotic therapy depended on the type and severity of infection, including but not limited to, quinolones (levofloxacin), cephalosporins (ceftriaxone, ceftazidime, cefixime, and cefoperazone tazobactam), beta-lactam antibiotics (amoxicillin, meropenem, and biapenem), and vancomycin. In addition to these treatments, patients with severe pneumonia or rapid deterioration received intravenous methylprednisolone (dose adjusted according to the disease state) for a median of 4 days (range: 2 - 11 days). The proportion of severe patients who received glucocorticoids was $60 \%$, which was significantly higher than that in non-severe patients (13.6\%). Moreover, severe patients received a significantly higher number of different types of drugs compared with non-severe patients (Table 1).

All patients in this study recovered and were discharged from hospital after a median hospitalization time of 15 days (range: 3 - 36 days). The hospitalization time for severe patients was significantly longer than for non-severe patients (23.0 days vs 12.5 days, $\mathrm{p}<0.001$ ).

\section{Discussion}

COVID-19 spread rapidly throughout the world, and infected people may progress quickly to acute respiratory distress syndrome, multisystem organ failure, and ultimately, death. In the absence of specific therapeutic drugs or vaccines for COVID-19, it is essential to detect the disease at an early stage and immediately isolate an infected person from the healthy population. Investigating the clinical features and risk factors regarding the progression of COVID-19 might help identify a new case and control disease spread.

In our study, people of all ages were susceptible to the virus (median: 54 years; range: 16 - 87 years), and $66 \%$ of the patients were $\geq 40$ years of age. No significant difference was found regarding the prevalence of COVID-19 by sex; however, middle-aged and older men may be more likely to develop severe infections. Other than decreasing innate immunity related to aging, the male sex difference may also relate to the influence of comorbidities, such as diabetes, hypertension, and coronary heart disease. Outbreaks of family clusters occurred with $43.8 \%$ of the patients, similar to findings in a previous report stating that per- 
son-to-person spread is a major route of transmission and indicating the importance of isolation [5]. Isolation at home, mobile cabin hospitals, and the 76-day lockdown of Wuhan City have been the most effective and immediate methods of controlling the spread of the $2019-\mathrm{nCoV}$ virus in China, by which, the COVID19 epidemic has been gradually controlled in China. The symptoms of SARSCoV-2 infection vary widely, from asymptomatic disease to pneumonia and life-threatening complications. Fever, cough, fatigue, and shortness of breath/ difficulty breathing are the most common symptoms, but other symptoms are diarrhea, anorexia, sore throat, nausea/vomiting, sore muscles, and other nonspecific symptoms. Some symptoms are similar to those seen with other respiratory illnesses, such as influenza, but it is important to identify and differentiate between these symptoms.

In addition to epidemiological histories and clinical manifestations, SARSCoV-2 RNA detection and chest imaging are the main measures in suspected cases to diagnose patients as confirmed cases. Among the overall population in our study, $83.8 \%$ of the patients had positive RNA detection test results and signs of pneumonia on CT scans at admission. Furthermore, 14 (10.8\%) patients had typical COVID-19 chest imaging features without confirmed positive RNA detection with RT-PCR from throat swab samples during hospitalization. With limitations of sample collection and transportation, as well as test kit performance, the total positive rate with RT-PCR using throat swab samples has been reported to be insufficiently sensitive, indicating that a large number of COVID19 patients will not be identified quickly. Unidentified infected patients may not receive appropriate treatment and carry a risk of infecting a larger population. Tao et al. [12] proposed that for early diagnosis of COVID-19, chest CT imaging may be a more reliable, practical, and rapid method of diagnosing and assessing COVID-19 than RT-PCR. The period of detectable 2019-nCoV RNA in our patient groups varied greatly (range: 1 - 41 days). To differentiate mild and moderate patients, chest radiograph offers an important diagnostic clue [13]. Typically, patients presented with unilateral, predominantly peripheral, areas of groundglass consolidation. After approximately 5 - 7 days, progressive multifocal peripheral air-space ground-glass consolidation or bilateral patchy consolidation, with diffuse ground-glass opacities were observed in patients with the severe form of COVID-19. These patients presented with emergent COVID-19 signs, such as trouble breathing, chest pain or pressure, and other hypoxemia-associated manifestations.

With disease progression, routine peripheral blood testing showed decreased lymphocytes, elevated white blood cell counts, neutrophils, and inflammatory markers (i.e., erythrocyte sedimentation rate, C-reactive protein, and serum amyloid A). Other studies found that several other inflammatory cytokines, such as granulocyte colony-stimulating factor, interferon gamma-inducible protein10 , monocyte chemoattractant protein-1, macrophage inflammatory protein-1A, and tumor necrosis factor- $\alpha$, were increased in patients with COVID-19 in the 
intensive care unit [14]. These findings suggest that cytokine storm is positively correlated with disease severity and may be related to an excessive inflammatory response in patients with severe COVID-19 and damage to innate and adaptive immunity [15].

Other affected organ systems such as the liver, cardiac system, kidneys, and coagulation system may be affected by cytokine storm and manifest as abnormal serum biochemical test results, especially in severe patients. A notable mechanism of this virus is that it spreads through the bloodstream and settles mainly in the lungs, as well the gastrointestinal tract, heart, and kidney, presumably concentrating in tissues that can express angiotensin-converting enzyme 2 , the receptor for SARS-CoV-2 [16].

Of the 130 patients with COVID-19 in our study, $21.5 \%$ presented with slightly abnormal ALT and/or AST levels, and 42.3\% had reduced serum albumin levels. Patients with severe COVID-19 seem to have higher rates of liver dysfunction. The pathogenesis of COVID-19-associated liver injury involves five main aspects: direct damage caused by the virus, immune-mediated stress and inflammation, hepatic ischemia and hypoxia, reactivation or aggravation of underlying liver disease, and drug hepatotoxicity [8]. We also observed that severe patients with pre-existing conditions (i.e., diabetes and hypertension) were more likely to develop liver injury. In contrast, few mild/moderate patients presented with abnormal or worsened liver function, regardless of comorbidities, such as fatty liver and chronic hepatis B. These findings are consistent with other clinical reports [14] [17] [18]. In the early management of COVID-19 in China, drug therapy always consisted of combined antiviral, antibiotic, and herbal therapies. As a result, drug-induced liver injury could not be differentially diagnosed. One patient in our study with ALT levels $>900 \mathrm{U} / \mathrm{L}$ was diagnosed with drug-induced liver injury after comprehensive analysis of his status and therapy. Therefore, recording the exact drugs administered and intensive surveillance of liver enzymes is necessary in the treatment of COVID-19 to identify liver injury early and prevent deterioration.

Abnormal myocardial enzyme levels (creatine kinase-MB, myoglobin, and cardiac troponin $\mathrm{T}$ ) or elevated $\mathrm{N}$-terminal B-type natriuretic peptide precursor were found in $16.9 \%$ of our patients. Additionally, $10.0 \%$ of patients developed kidney dysfunction measured by urea and serum creatinine levels, and estimated glomerular filtration rate. Coagulation dysfunction manifested mainly as elevated D-dimer levels, which was seen in $24.6 \%$ of the patients. Damage to these organs and systems may be ascribed to anoxia and hypotension secondary to the inflammatory cytokine storm activated by SARS-CoV-2. Moreover, intestinal dysbacteriosis and endotoxemia in patients with severe COVID-19 can also influence liver and coagulation function. Organ system injuries, especially parenchymal cells within the organs, can also be aggravated by hypoalbuminemia. Therefore, myocardial, kidney, and coagulation function should be closely monitored when managing patients with COVID-19. 
Apart from oseltamivir and arbidol in our study, the most common antiviral drug was Lianhua Qingwen. Compound herbal remedies based on "syndrome differentiation" were also used as combination therapy in some patients. Patients with increased white blood cell numbers in peripheral blood or with suspected bacterial infection were treated with moxifloxacin and third-generation cephalosporins. Patients without liver injury could be treated with azithromycin, which may prevent bacterial superinfection and which has immunomodulatory properties, as adjunct therapy [19]. These pharmacological therapies effectively prevented disease progression, in our study. Methylprednisolone was administered in patients with overly exuberant systemic inflammatory responses, to prevent rapid progression to acute respiratory disease syndrome and alleviate the related signs [20]. In this study, $60 \%$ of severe patients were treated with corticosteroids for a short period of time ( 3 - 12 days), and all patients were discharged in better health. However, corticosteroid therapy is not recommended for viral pneumonia and is controversial, with no general agreement regarding its clinical use, in patients with COVID-19. Using corticosteroids as short-term, low-dose therapy is considered ideal, to avoid adverse effects such as bacterial infection and prolonged viral clearance time. With these treatments and close surveillance, all 130 of our patients were discharged from hospital with significant improvement.

\section{Conclusion}

In conclusion, as a new acute respiratory infectious disease, COVID-19 has such a high efficiency of interpersonal transmission that clustered occurrences easily develop in people without protection. Middle-aged and older men with pre-existing conditions are more likely to develop severe COVID-19. Multiple organs or systems may be involved in the disease, and timely treatment is needed to prevent disease progression to life-threatening levels. With the global COVID-19 pandemic, the causative virus' pathogenesis, clinical characteristics of different populations, and effective therapeutic strategies need to be clarified further in multicenter and large population-based clinical studies.

\section{Funding}

The authors disclosed receipt of the following financial support for the research, authorship, and/or publication of this article: Hebei Provincial Department of Science and Technology [Grant number: 20277713D].

\section{Conflicts of Interest}

The authors declare no conflicts of interest regarding the publication of this paper.

\section{References}

[1] Wu, F., Zhao, S., Yu, B., Chen, Y.M., Wang, W., Song, Z.G., et al. (2020) A New Coronavirus Associated with Human Respiratory Disease in China. Nature, 579, 265 
269. https://doi.org/10.1038/s41586-020-2008-3

[2] World Health Organization (2020) Coronavirus Disease (COVID-19) Dashboard. https://covid19.who.int/

[3] Morawska, L. and Cao, J.J. (2020) Airborne Transmission of SARS-CoV-2: The World Should Face the Reality. Environment International, 139, Article ID: 105730. https://doi.org/10.1016/j.envint.2020.105730

[4] Wang, D.W., Hu, B., Hu, C., Zhu, F.F., Liu, X., Zhang, J., et al. (2020) Clinical Characteristics of 138 Hospitalized Patients with 2019 Novel Coronavirus-Infected Pneumonia in Wuhan, China. The Journal of the American Medical Association, 323, 1061-1069. https://doi.org/10.1001/jama.2020.1585

[5] Henry, B.M. and Vikse, J. (2020) Clinical Characteristics of Covid-19 in China. The New England Journal of Medicine, 382, 1859-1862. https://doi.org/10.1056/NEJMc2005203

[6] Chen, N., Zhou, M., Dong, X., Qu, J.M., Gong, F.Y., Han, Y., et al. (2020) Epidemiological and Clinical Characteristics of 99 Cases of 2019 Novel Coronavirus Pneumonia in Wuhan, China: A Descriptive Study. Lancet, 395, 507-513. https://doi.org/10.1016/S0140-6736(20)30211-7

[7] National Health Commission of the People's Republic of China and Traditional Chinese Medicine of the People's Republic of China (2020) Guidelines for the Diagnosis and Treatment of Coronavirus Disease 2019 (Trial Version 7). http://www.nhc.gov.cn/yzygj/s7653p/202003/46c9294a7dfe4cef80dc7f5912eb1989.s $\underline{\mathrm{html}}$

[8] Chinese Digestion Association, Chinese Medical Doctor Association, Chinese Society of Hepatology, Chinese Medical Association (2020) The Protocol for Prevention, Diagnosis and Treatment of Corona Virus Infective Disease 2019. Chinese Journal of Hepatology, 28, 217-221.

[9] The Study Group of Drug Induced Liver Disease of Chinese Medical Association (2015) Diagnosis and Treatment Guideline on Drug-Induced Liver Injury. Chinese Journal of Hepatology, 23, 810-820.

[10] Khwaja, A. (2012) KDIGO Clinical Practice Guideline for Acute Kidney Injury. Nephron Clinical Practice, 120, c179-c184. https://doi.org/10.1159/000339789

[11] National Center for Gerontology/National Clinical Research Center for Geriatric Disorders, Cardiovascular Branch of Chinese Geriatrics Society, Imaging Group of Cardiovascular Department, Beijing Medical Association (2020) Expert Recommendations for Clinical Management of Myocardial Injury Associated With Coronavirus Disease 2019 (First Edition). Chinese Circulation Journal, 35, 326-330.

[12] Ai, T., Yang, Z.L., Hou, H.Y., Zhan, C.A., Chen, C., Lv, W.Z., et al. (2020) Correlation of Chest CT and RT-PCR Testing in Coronavirus Disease 2019 (COVID-19) in China: A Report of 1014 Cases. Radiology, 296, E32-E40.

https://doi.org/10.1148/radiol.2020200642

[13] Bernheim, A., Mei, X.Y., Huang, M.Q., Yang, Y., Fayad, Z.A., Zhang, N., et al. (2020) Chest CT Findings in Coronavirus Disease-19 (COVID-19): Relationship to Duration of Infection. Radiology, 295, 685-691. https://doi.org/10.1148/radiol.2020200463

[14] Huang, C.L., Wang, Y.M., Li, X.W., Ren, L.L., Zhao, J.P., Hu, Y., et al. (2020) Clinical Features of Patients Infected with 2019 Novel Coronavirus in Wuhan, China. Lancet, 395, 497-506. https://doi.org/10.1016/S0140-6736(20)30183-5

[15] Ye, Q., Wang, B.L. and Mao, J.H. (2020) The Pathogenesis and Treatment of the 'Cytokine Storm' in COVID-19. The Journal of Infection, 80, 607-613. 
https://doi.org/10.1016/j.jinf.2020.03.037

[16] Chai, X.Q., Hu, L.F., Zhang, Y., Han, W.Y., Lu, Z., Ke, A.W., et al. (2020) Specific ACE2 Expression in Cholangiocytes May Cause Liver Damage After 2019-nCoV Infection. BioRxiv, Article ID: 931766. https://doi.org/10.1101/2020.02.03.931766

[17] Guan, W.J. and Zhong, N.S., et al. (2020) Clinical Characteristics of 2019 Novel Coronavirus Infection in China. The New England Journal of Medicine, 382, 1708 1720. https://doi.org/10.1056/NEJMoa2002032

[18] Zhang, C., Shi, L. and Wang, F.S. (2020) Liver Injury in COVID-19: Management and Challenges. The Lancet: Gastroenterology \& Hepatology, 5, 428-430. https://doi.org/10.1016/S2468-1253(20)30057-1

[19] Zarogoulidis, P., Papanas, N., Kioumis, I., Chatzaki, E., Maltezos, E. and Zarogoulidis, K. (2012) Macrolides: From in Vitro Anti-Inflammatory and Immunomodulatory Properties to Clinical Practice in Respiratory Disease. European Journal of Clinical Pharmacology, 68, 479-503. https://doi.org/10.1007/s00228-011-1161-x

[20] Zhou, Y.H., Qin, Y.Y., Lu, Y.Q., Sun, F., Yang, S., Harypursat, V., et al. (2020) Effectiveness of Glucocorticoid Therapy in Patients with Severe Novel Coronavirus Pneumonia: Protocol of a Randomized Controlled Trial. Chinese Medical Journal, 133, 1080-1086. https://doi.org/10.1097/CM9.0000000000000791

\section{Abbreviations}

COVID-19: Coronavirus Disease 2019

SARS-CoV-2: Severe Acute Respiratory Syndrome Coronavirus 2

RT-PCR: Real-Time Reverse Transcription Polymerase chain Reaction 2019-nCoV: 2019-Novel Coronavirus

ALT: Alanine Aminotransferase

AST: Aspartate Aminotransferase 